arguments about these. Whereas one would like to advocate layer upon layer of rules and regulations, the other prefers further education and open debate about all these issues so that a collective decision can be made. Stock's 'Appendix 2' with eight hypothetical scenarios of genetic manipulation is a starting point to test one's own feelings and thoughts about these issues and to initiate discussion around them. Fukuyama gives us no such opportunity. He just tells us what should be done.

In my opinion, read Fukuyama's Doomsday warnings, but be aware of his trappings; and read Stock's concerns in order to re-think your own opinions. Creating fear only benefits those few who have some other personal and political agenda to follow. And by the way, my Greek friends tell me that if I want to coin the term for the fear of the future, it could be 'mellontophobia', which, like most other phobias, is not a desirable state of mind. Fukuyama frightens, Stock stimulates!

Suresh I.S. Rattan is at the Danish Centre for Molecular Gerontology at the University of Aarhus, Denmark.

E-mail: rattan@imsb.au.dk

DOI: 10.1093/embo-reports/kvf209

\section{Lucky experiment}

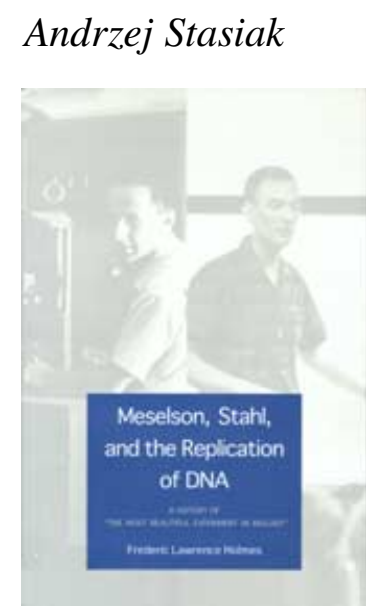

Meselson, Stahl, and the Replication of DNA by Frederic Lawrence Holmes

Yale University Press, New Haven, CT, USA

510 pages, $€ 55.00$

ISBN 0300085400

The 1957 Meselson-Stahl experiment is known to be so logical and so perfect that little effort is required to recall its design and its outcome. But is it worth reading another 500 pages about it, when it is already described in every biology textbook? I believe so. Frederic Lawrence Holmes, Professor of History of Medicine at Yale University, spent more than 10 years researching and writing this book, and the end result is not only highly enjoyable but also holds quite a surprise. Matthew Meselson and Frank Stahl shared their memories with Holmes and allowed him access to their laboratory journals and other documents of the period; additional illuminations are provided by interviews with other prominent scientists of the time, such as Jim Watson and John Cairns.

Every biologist is familiar with the elegant demonstration of the semiconservative mechanism of DNA replication. To produce labelled DNA of high buoyant density, bacteria were grown for several generations in a medium containing $\mathrm{N}^{15}$ compounds as a nitrogen source. Upon transfer to the 'light' medium containing $\mathrm{N}^{14}$, Meselson and Stahl observed that the first round of DNA replication produced molecules with an intermediate density corresponding to that predicted for those composed of one heavy and one light strand. The second round produced a 1:1 mixture of light and intermediate density
DNA molecules. DNA molecules with one of these three buoyant densities could be separated and formed clearly defined bands in a caesium chloride density gradient. This very clean, clear-cut result supported the then much-debated proposal made in 1953 by Watson and Crick, which stated that, during replication, the two strands of DNA separate and each serves as a template for the newly synthesised complementary strands. In fact, the Meselson-Stahl experiment made its prominent entry into the history of science since it is considered to be the first experimental proof of the Watson and Crick DNA model.

For me, the book has two culminating points, although I am not sure that the author intended the second. The first highlight of the book comes after the very slow, somewhat random progress of the Meselson and Stahl joint project, suddenly everything starts working correctly and, following a series of analytical ultracentrifuge runs, Meselson develops a film showing three well-defined DNA bands. The second highlight, which was much more thrilling for me, was the realisation that the experiment only worked due to a fortuitous artefact.

Now that we know much more about the replication of bacterial chromosomes, we may ask whether the experimental design of Meselson and Stahl should have produced the result that they obtained. We know that bacterial chromosomes, replicate continuously during logarithmic growth and also that a new round of replication starts even before the previous one is completed. Meselson and Stahl did not synchronise the bacteria they worked with; therefore, when the culture was transferred from the heavy to the light medium, the chromosomes of each cell were replicated to a different extent. Consequently, when the first sample was analysed after 0.3 of a generation time of growth in the light medium, molecules that had just finished replication should have diminished their buoyant density to a similar extent as those that had simply advanced in their replication cycle, since both these categories should have incorporated similar proportions of bases containing $\mathrm{N}^{14}$. Instead, Meselson and Stahl observed that $\sim 30 \%$ of the DNA molecules 'jumped' to the clearly defined intermediate density level, while the remaining $70 \%$ stayed at an unchanged high density level. At subsequent time 


\section{books}

points, they observed the prompt appearance of an increasing number of DNA molecules with the intermediate density followed by the appearance of the low density DNA molecules. In actual fact, as the replication of $\mathrm{N}^{15}$-labelled chromosomal DNA proceeded in the light medium, a progressive, continuous change of DNA density of the replication intermediates should have been observed. Had Meselson and Stahl detected such a continuous change of DNA density they would have probably interpreted it as proof of the distributive mode of DNA replication where frequent strand breakages and rejoining had been proposed to circumvent the need to unwind the double helix. Such a model was strongly favoured by Max Delbrück, Meselson's mentor at Caltech, who had challenged and encouraged him to perform an experiment that could distinguish between the two modes of replication.
How is it therefore possible that the Meselson-Stahl experiment resulted in DNA bands of the three 'quantized' buoyant densities? The answer lies in the fortuitous and not then recognised artefact caused by the shearing of the DNA during its passage through the hypodermic needle used to fill the ultracentrifuge tubes. In fact, Meselson and Stahl did not even consider the possibility of DNA fragmentation, since in the same, memorable PNAS paper they measured the thickness of the observed DNA bands in order to determine the native molecular weight of bacterial DNA. Biology also helped them obtain their clean result, since there is only one origin of DNA replication per bacterial chromosome, which also was not known at the time. Fragmentation of partially replicated bacterial chromosomes into approximately 400 pieces caused the great majority of these fragments to consist of strands that were either entirely heavy or entirely light. Fragments that had a strand with a transition point from heavy to light and would thus have 'nonquantized' density would have been very rare (1 per 200) and thus not visible in the caesium chloride density gradient. Thanks to this artefact, Meselson and Stahl obtained the results we all know now from textbooks. Amazingly, the accidental fragmentation of the DNA was just as it should be. Smaller fragments would have formed very diffuse bands in the caesium chloride gradient, precluding the distinction between different density species and giving an impression of a continuous density distribution also supporting the distributive mode of DNA replication. Holmes subtitles his book as 'a history of the most beautiful experiment in biology'. He should add that it is also probably the luckiest.

Andrzej Stasiak is at the University of Lausanne, Switzerland.

E-mail: Andrzej.Stasiak@lau.unil.ch

DOI: $10.1093 /$ embo-reports/kvf210 